Rajan K Sharma, MS

Department of Neurosurgery

Bir Hospital, NAMS

Kathmandu, Nepal

Gopal R Sharma , MS

Department of Neurosurgery

Bir Hospital, NAMS

Kathmandu, Nepal

Prakash Bista, MCh

Department of Neurosurgery

Bir Hospital, NAMS

Kathmandu, Nepal

Rajiv Jha, MCh

Department of Neurosurgery

Bir Hospital, NAMS

Kathmandu, Nepal.

Address for Correspondence:

Rajan K Sharma, MS

Department of Neurosurgery

Bir Hospital, NAMS

Kathmandu, Nepal

Email: rajanktm58@gmail.com

Received, 22 June, 2017

Accepted, 9 July, 2017

$\mathrm{R}$ oberts syndrome is a rare autosomal recessive genetic disorder. It carries the name of John Roberts, who first reported the case of a male infant with bilateral cleft lip and tetraphocomelia in 1919. ${ }^{7}$ The combination of malformations was recognized as a syndrome in 1966 by Appelet and coworkers. Hermann et al. reported similar but milder malformations which were referred to as 'pseudothalidomide SC syndrome' in 1969. These two syndromes had varying phenotypic expression and were later concluded as the same entity because of resemblance of thalidomide embryopathy with Roberts syndrome and were therefore termed as Roberts SC phocomelia syndrome. ${ }^{10}$ In 1995, two Colombian geneticists Hugo and Vega discovered the Roberts gene, called ESCO2 gene which is located at 8p21.1. ${ }^{11}$ Typical clinical features of Roberts syndrome are pre-natal and postnatal growth retardation, bilateral symmetric limb reduction and craniofacial abnormalities. ${ }^{10}$ This syndrome is rare with approximately 100 cases described in the literature and only a single case reported from Pakistan in $1993.3,9$

\section{A Child with Roberts Syndrome: A Case Report}

Roberts syndrome is a genetically determined rare birth defect causing, skeletal deformities, particularly symmetrical limb reduction and craniofacial anomalies. For any child with limb and craniofacial bony malformations, this syndrome should be considered in the differentials. Although this syndrome represents only a small proportion of the total number of individuals with limb deficiency, it is important to be identified in order to give accurate genetic counseling including recurrence risk in siblings and possible prenatal diagnosis. This is the case report of a 9 year old female child who presented with defective development of all four extremities and craniofacial abnormalities. The overall clinical and radiological features were suggestive of Roberts syndrome.

Key Words: autosomal recessive, Roberts SC phocomelia syndrome, tetraphocomelia

Roberts syndrome is also known by many other names, including: Hypomelia-Hypotrichosis-Facial Hemangioma Syndrome, SC Syndrome (once thought to be an entirely separate disease), Pseudothalidomide Syndrome, RobertsSC Phocomelia Syndrome, SC Phocomelia Syndrome, Appelt-Gerken-Lenz Syndrome, RBS, SC Pseudothalidomide Syndrome, and Tetraphocomelia-Cleft Palate Syndrome.

\section{Case Report}

We present here the case of a 9-year-old girl, born to nonconsanguinous parents, from the province of Lalitpur was admitted via Neurosurgical OPD with complaint of bilateral upper and lower limb abnormalities and abnormal growth of head. The first child of the parents is twenty five years old lady, is normal and was born after nine months of intrauterine life. The second conception is 23 years old boy. She was born to a 36 year old mother and 45 year old father and there was no consanguinity. The girl was the fourth child born via spontaneous vaginal delivery at 


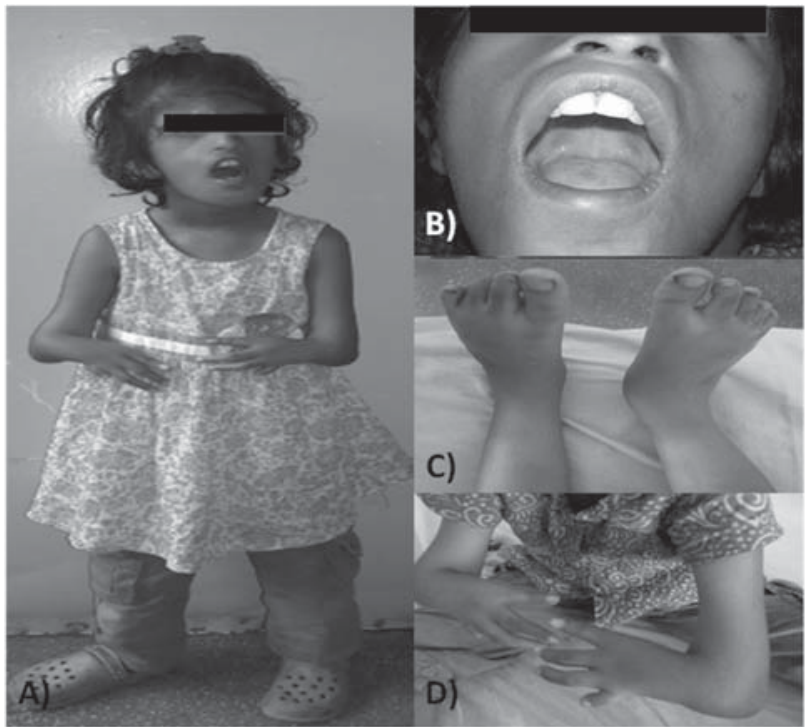

Figure 1: Clinical findings, A) A 9-year-old girl with Short stature, microbrachycephaly, phocomelia and absence of thumb in bilateral upper limbs, B) facial picture showing micrognathia, malar hypoplasia, downslanting palpebral fissure, ocular hypertelorism, corneal clouding, beaked nose, hypoplastic nasal alae etc, C) oligodactyly and absence of thumb with contracture at wrist joint, D) oligodactyly in left foot and right foot appears normal

hospital and had no history of birth asphyxia. Both parents were healthy. There was no history of any congenital malformation in family. There was history of fever in antenatal period during second trimester of pregnancy which was for around one month and was treated with antibiotics and analgesics. There was no history of other illness like rash, hypertension and no teratogens exposure was noted during pregnancy except drugs taken during pregnancy. There was no history of abortion or still birth. The child had normal head developmental parameters with holding seen at two and a half months. At the age of 9 years she is studying in class one.

On presentation the growth parameters were below $4^{\text {th }}$ centiles; weight was $14 \mathrm{~kg}$, length was $104 \mathrm{~cm}$ and the head circumference was $41 \mathrm{~cm}$. The patient had characteristic dysmorphic facies with defective development of all four extremities that was the main constituent of malformation complex.The craniofacial abnormalities included prominent frontal bones, microbrachycephaly, small low set ears, prominent frontal bones, prominent eyes, shallow orbits, hypertelorism, hypoplastic ala nasai, micrognathia, short neck and down slanting palpebral fissures (Figure 1).

Head circumference $41 \mathrm{~cm}$ (expected $52 \mathrm{~cm}$ ), lower limb: upper limb ratio 47:30 (expected 1:1), and mid arm circumference $12 \mathrm{~cm}$ (expected $>14 \mathrm{~cm}$ ). An X-ray of the

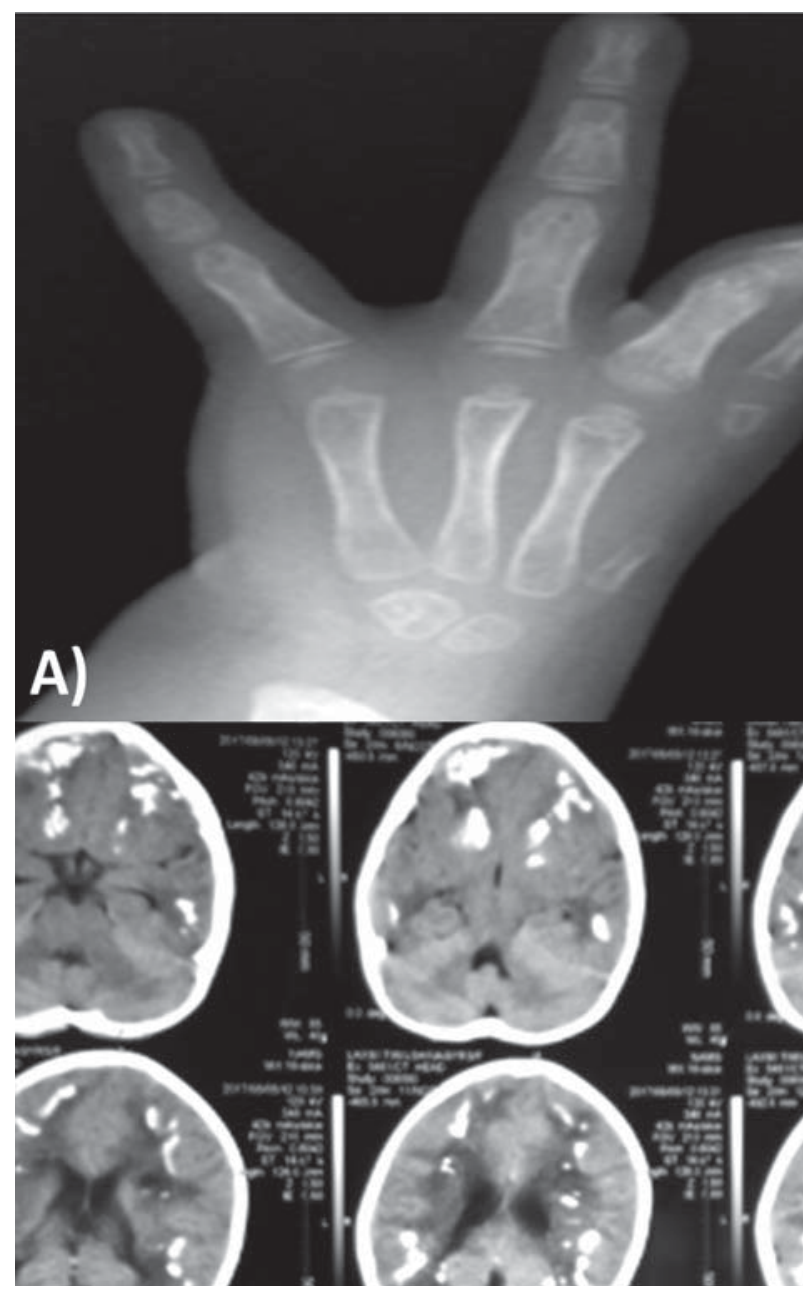

Figure 2: Radiological images, A) X-ray of hand showing oligodactyly and absence of thumb, CT Scan axial view of brain showing multiple hyperdense lesion likely calcification which is in tram track pattern and can be due to intrauterine infection

upper limbs showed symmetrical ulnar bone shortening on both sides, the ulna measuring $9 \mathrm{~cm}$ (expected 13.15 $\mathrm{cm}$ ) (Figure 2 A). On right leg she had five phalanges but on left she had four phalanges. There was symmetrical shortening of the tibia by $11 \mathrm{~cm}$ (Right) and $11.5 \mathrm{~cm}$ (Left) (expected 22.5 to $24 \mathrm{~cm}$ ). Dental examination revealed a few hypodontic teeth in the oral cavity.

She had severe fixed flexion deformities of all limbs. The limbs were short with hands and feet located closed to the body. The thumbs were absent bilaterally, with oligodactyly of upper and lower extremities and flexion deformity at wrist joints. In both eyes there was proptosis and whitish hue of cornea. She could fix and follow objects. There was no visceromegaly and no cardiovascular defects.

Radiographic examination showed agenesis of radii and ulna, absence of first metacarpal bilaterally. Pelvis and 
iliac bones were normal; there was bilateral shortening of tibia. Other tests including echocardiography and abdominal ultrasound were normal.

Complete blood count was normal with no thrombocytopenia. Cytogenetic studies could not be done due to unavailability of facilities. Diagnosis of Roberts syndrome was made on the basis of craniofacial malformations and limb deformities (tetraphocomelia) and growth retardation. CT Scan axial view of brain showed multiple hyperdense lesion likely calcification which is in tram track pattern and may be due to intrauterine infection (Figure 2 B).

\section{Discussion}

Roberts Syndrome is a very rare congenital malformation. Its prevalence is unclear. Thus far, about 150 cases of different racial and ethnic backgrounds have been reported in the literature. ${ }^{4}$ The major abnormalities required to make the diagnosis of Roberts Syndrome include: mental retardation, growth retardation with prenatal onset and continuing postnatally, midline craniofacial abnormalities, tetra-hypomelia that are more prominent in the upper extremities varying from phocomelia to simple shortness of the extremity and accompanying extremity abnormalities. ${ }^{4,11}$ In 2005, the disease gene was determined by Vega et al. ${ }^{11}$ All of the above findings were determined in our case except genetic analysis which was not done due to unavailability.

The craniofacial and extremity abnormalities seen in this syndrome include cleft lip and palate, craniosynostosis, microcephalia, silver-coloured or sparse hair, micrognathia, ocular hypertelorism, exophthalmia, eyelid coloboma, corneal blurring, cataract, mid-facial capillary haemangioma, ear malformations, low-set ears, maxillary protrusion, malar hypoplasia, hypoplastic mandible, nasal hypoplasia and short neck bilateral symmetrical tetraphocomelia or hypomelia, brachydactyly/oligodactyly. ${ }^{1}$

In Roberts Syndrome, the upper extremities are affected more frequently than the lower extremities. In our case too we had upper extremities more involved then lower extremities. Cardiac anomalies are observed in about $50 \%$ of the cases. Atrial septal defect, ventricular septal defect, patent ductus arteriosus have previously been reported. ${ }^{5}$ However, valvular aortic stenosis has not been mentioned in the literature. In our case there was no cardiac problem. Other less common features are renal anomalies (polycystic, dysplastic kidneys), cryptorchidism, neurological anomalies like microcephaly and hydrocephalus; and femoral tibial ankylosis. The reasons for death of these patients are generally cardiac, renal anomalies and infections.
Chromosomal anomalies such as premature centromere separation [PCS, heterochromatin push] and/or ESCO2 mutations on the 8th chromosome are determined on cytogenetic evaluation and molecular studies. ${ }^{4,11}$ Despite the observation of PCS as a characteristic chromosomal finding in most of the RBS cases, there are also some reported cases with normal chromosomes. Most patients born with growth retardation, severe craniofacial and limb defects have died early in childhood. Those with less severe defects have better prognosis. Aggressive medical intervention that is; correcting cleft lip and palate, correcting orthopaedic deformities and nutritional rehabilitation is suggested along with parental counselling. ${ }^{2}$

The unique cytogenetic abnormality called premature centromere separation which disrupts the process of chromatid pairing, is responsible for the development of multiple structural anomalies found in Robert SC Syndrome. Premature centromere separation has been reported in lymphocytes and/or fibroblasts from patients whose clinical phenotypes cover the range of the Roberts syndrome, from a severe variant to SC phocomelia syndrome at the milder end. ${ }^{8}$ Jabs et al. ${ }^{6,8}$ presented evidence that Roberts syndrome is a mitotic mutant. They emphasized that, in addition to previously described changes, aneuploidy with random chromosome loss and micronuclei and/or nuclear lobulation in the interphase cells are characteristic features of this syndrome. They suggested however, that the defect might lie in one of the proteins transiently associated with the kinetochore being involved in its function.

Stioui et al. detected premature centromere separation on chorionic villus sampling at eight weeks. gestation in a woman at a risk of recurrence of Roberts syndrome. Hirschhorn and Kaffe pointed out that they had made a prenatal diagnosis of Roberts-SC syndrome in a family at risk by detection of skeletal and renal abnormalities.

\section{References}

1. Abbas R, Waqar S, Ahmad TM et al. A Child with Roberts Syndrome. J Coll Physicians Surg Pak 21: 431-433,2011

2. DaSilva EO, Bezerra LH. The Roberts syndrome. Hum Genet 61: 372-374,1982

3. Gordillo M, Vega H, Trainer AH et al. The molecular mechanism underlying Roberts syndrome involves loss of ESCO2 acetyltransferase activity. Hum Mol Genet 17: 2172-2180, 2008

4. Gordillo M, Vega H, Jabs EW. Roberts Syndrome. In: Pagon RA, Adam MP, Bird TD, Dolan CR, Fong CT, Stephens K, editors. GeneReviews ${ }^{\mathrm{TM}}$ [Internet]. Seattle (WA): University of Washington, Seattle 


\section{Sharma et al}

2006; (Online) updated 2009 Apr 14 (Cited 2013 Dec 30). Available from URL: http://www.ncbi.nlm.nih. gov/books/NBK1153/.

5. Holden KR, Jabs EW, Sponseller PD. Roberts/ pseudothalidomidesyndrome and normal intelligence: approaches to diagnosis and management. Dev Med Child Neurol 34: 534-538, 1992

6. Jabs EW, Tuck-Muller CM, Cusano R et al . Studies of mitotic and centromeric abnormalities in Roberts syndrome: Implications for a defect in the mitotic mechanism. Chromosome 100: 251-252, 1991.

7. Murthy J, Dewan M, Hussain A. Roberts - SC syndrome, a rare syndrome and cleft palate repair, a case report. Indian J Plast Surg 14 : 222-225, 2008
8. Parry DM, Mulvihill JJ, Tsai S et al. SC phocomelia syndrome, premature centromere separation and congenital cranial nerve paralysis in two sisters, one with malignant melanoma. Am J Med Genet 24: 653-672,1986

9. Qazi A, Qazi SA, Khan MA. Roberts syndrome. J Pak Med Assoc 43: 164-166,1993

10. Temtamy SA, Ismail S, Helmy NA. Roberts syndrome: study of four new Egyptian cases with comparison of clinical and cytogenetic findings. Genet Couns 17:11-13, 2006

11. Vega H, Waisfisz Q, Gordillo $M$ et al. Roberts syndrome is caused by mutations in ESCO2, a human homolog of yeast ECO1 that is essential for the establishment of sister chromatid cohesion. Nat Genet 37: 468-70, 2005 